\title{
La interpretación bilateral en el ámbito turístico: evaluación
}

\author{
Carmen Álvarez García \& Christiane Limbach \\ Universidad Pablo de Olavide \\ carmenalvarez@upo.es \& clim@upo.es \\ https://dx.doi.org.10.12795/futhark.2019.il4.01
}

Fecha de recepción: 5 .10.2018

Fecha de aceptación: 10.02.2019

Resumen: A partir de la matriz formativa para evaluar la interpretación consecutiva de Domínguez Araújo (2013) hemos elaborado una rúbrica específica para evaluar la interpretación bilateral y la hemos aplicado a un corpus de interpretaciones bilaterales con la intención de diseñar estrategias didácticas útiles en la formación en este tipo de interpretación. La evaluación del corpus, compuesto por 14 exámenes finales de interpretación bilateral en el turismo con la combinación lingüística alemán-español, ha permitido categorizar los elementos en los que se cometen con mayor frecuencia los errores que impiden la producción de una interpretación adecuada. Los resultados son positivos en cuanto a la expresión en lengua materna y en la resolución de problemas. No obstante, constatan la necesidad de establecer nuevas estrategias didácticas que busquen mejorar la expresión en la lengua extranjera. Además, hemos podido comprobar que los errores más frecuentes de los estudiantes son las omisiones de información relevante y los cambios de sentido respecto al mensaje original. El análisis muestra que la rúbrica elaborada para la evaluación de interpretaciones bilaterales puede ser útil tanto para la gestión y diseño de estrategias didácticas como para potenciar el autoaprendizaje por parte de los estudiantes.

Palabras clave: interpretación bilateral, rúbrica de evaluación, corpus, formación en interpretación

\section{Bilateral Interpreting in the Touristic Field: Assessment}

\begin{abstract}
Based on the assessment rubric for consecutive interpretation, by Domínguez Araújo (20I3), we have elaborated a specific rubric for the assessment of bilateral interpretation that has been applied to a corpus formed by bilateral interpretations, in order to design didactic strategies useful for training in this kind of interpretation. The evaluation of the 14 final exams on bilateral interpretation in

Futhark I4 (2019)
$3-26$
\end{abstract}


the touristic field (German-Spanish), included in the corpus, allows the categorisation of those elements where errors more frequently occur preventing adequate interpretations. Results show that students perform well in expression in their mother tongue and in problem solving. They highlight, however, the needs for implementing new didactic strategies to improve the expression in the foreign language. Often, too, students omit relevant information from the original text or introduce changes in meaning. The analysis shows that the rubric elaborated to assess bilateral interpretations can be a useful tool for managing and designing didactic strategies as well as a self-learning tool for students.

Keywords: bilateral interpreting, assessment rubric, corpus, interpreting training

Sumario: Introducción. I. Metodología del análisis. 2. Descripción del corpus. 3. Resultados del análisis. Conclusiones

\section{Introducción}

La interpretación bilateral, también llamada de enlace o dialógica, consiste en la traslación de un mensaje entre dos intervinientes, por lo que cuenta con una característica especial que la diferencia del resto de interpretaciones: el esfuerzo de bidireccionalidad (Collados Aís y Fernández Sánchez, 200I: 66). Esta necesidad de interpretar hacia y desde la lengua materna conlleva una serie de dificultades particulares que no se dan en otros tipos de interpretación. Por ello, se incluye en la Universidad Pablo de Olavide (Sevilla, España), en el último curso del Grado en Traducción e Interpretación y en el Doble Grado en Humanidades y Traducción e Interpretación, una asignatura obligatoria dirigida a la formación específica en interpretación bilateral con la combinación lingüística de español con la primera lengua extranjera (inglés, francés o alemán), así como la posibilidad de cursar la asignatura optativa de interpretación bilateral en la segunda lengua extranjera.

En este trabajo, presentamos los primeros resultados de un análisis que hemos llevado a cabo sobre un corpus de interpretaciones bilaterales en el ámbito del turismo, con la combinación alemán-español-alemán, formado a partir de las producciones llevadas a cabo en el examen final de la asignatura Interpretación Bilateral $\mathrm{C} / \mathrm{A} / \mathrm{C}$, alemán, por alumnos con alemán como segunda lengua extranjera. Se ha puesto especial interés en la producción en la lengua extranjera para analizar los aspectos en los que el grupo de alumnos ha tenido más dificultades, así como aquellos en los que ha producido un resultado adecuado tras haber cursado el cuatrimestre completo. 
Entre los estudios publicados recientemente sobre enseñanza y evaluación de la interpretación, podemos destacar Cirillo y Niemants (2017), Aguirre Fernández-Bravo y Roca Urgorri (2015) y Aguirre Fernández-Bravo (2016) y, en el ámbito de la interpretación social, Ruiz Mezcua (2010). La mayoría toma una perspectiva didáctica, con propuestas formativas muy interesantes, diseñadas para conseguir la competencia interpretativa. Si bien, no son tan abundantes los estudios que abordan la evaluación de la interpretación y los que hay se concentran en tipos de interpretación que difieren de la dialógica o bilateral. Por esta razón, decidimos realizar nuestro análisis a partir de la rúbrica de interpretación consecutiva elaborada por Domínguez Araújo (20/3), que la autora denomina matriz, para adaptarla a la interpretación bilateral mediante la inclusión de categorías adicionales, propias de este tipo de interpretación.

Con nuestro trabajo, pretendemos contribuir, en la medida de nuestras posibilidades, a la formación en interpretación bilateral en el ámbito del turismo, analizando un aspecto que hemos visto que no se ha abordado lo suficiente y que nos parece de gran relevancia para la docencia, como es la evaluación de la producción. De esta manera, hemos optado por emplear una rúbrica de evaluación, ya que, aunque no es frecuente su empleo en la educación superior, se trata de una «herramienta especialmente útil para guiar procesos de evaluación y generar una información de calidad sobre sus resultados» (Alcón Latorre, 2016: I).

Reddy y Andrade (2009) indican que, en una rúbrica de evaluación, los criterios deberán establecerse de manera previa al proceso evaluador y contendrán la descripción de los elementos importantes en la evaluación, de tal manera que sirvan para establecer el grado de calidad del trabajo mediante una escala ascendente que muestre distintos niveles de consecución de las competencias o habilidades requeridas (Reddy y Andrade, 2009: 435). De igual manera, las autoras destacan que, para autores como Arter y McTighe (200I) o Stiggins (200I), los estudiantes también pueden emplear las rúbricas para evaluar el progreso de su aprendizaje (Reddy y Andrade, 2009: 437).

La rúbrica diseñada se ha aplicado a las producciones de exámenes de la asignatura Interpretación Bilateral $\mathrm{C} / \mathrm{A} / \mathrm{C}$ alemán, que es optativa y está dirigida a aquellos estudiantes con alemán como segunda lengua extranjera, por lo que concentra a un número reducido de alumnos (entre 10 y 12 ). Se imparte en el segundo cuatrimestre del último curso y se configura en tres bloques correspondientes a distintos ámbitos donde se suelen requerir con frecuencia servicios de interpretación bilateral. Entre dichos ámbitos, se encuentra el turismo, motor económico en la comunidad autónoma de Andalucía, que genera potenciales puestos de trabajo para nuestros egresados.

El análisis llevado a cabo parte de tres objetivos fundamentales: 
a) desarrollar una rúbrica de evaluación específica para la interpretación bilateral que incluya aquellos elementos que generan dificultades a la hora de producir una interpretación bilateral adecuada, como los números, las fechas, los precios, etc., especialmente en la combinación alemán-español, así como referencias culturales, que suelen darse con mucha frecuencia en el ámbito turístico (procedentes por ejemplo de la gastronomía, la geografía, los monumentos, etc.)

b) identificar y clasificar los errores producidos en las interpretaciones de examen

c) desarrollar estrategias didácticas que mejoren la adquisición de competencias por parte de los alumnos a la hora de realizar interpretaciones bilaterales en el ámbito del turismo.

\section{Metodología del análisis}

La tabla I muestra la matriz formativa para evaluar la interpretación consecutiva, elaborada por Domínguez Araújo (2013), donde se establecen cuatro áreas a considerar en la evaluación de la interpretación consecutiva: la presentación, la expresión, la resolución de problemas y el contenido. Para estas cuatro áreas se establece una puntuación que varía de 0 a una puntuación máxima de 5, con un requisito específico por el que el área 'contenido' debe aprobarse con un mínimo de 2,5 puntos para poder aprobar el examen. Este elemento evaluativo del contenido se divide a su vez en dos categorías, de tal manera que se evalúa por un lado la síntesis y la división de la atención y, por otro lado, la equivalencia. Esta diferenciación también se produce en la puntuación que se le otorga a la producción, pues mientras que, en el primer elemento, la puntuación sigue empleando el rango de 0 a 5 , la equivalencia solo suma I punto en caso de que no se agregue ninguna idea no incluida en el mensaje original, exceptuando las explicaciones necesarias sobre elementos culturales, y puede valorarse con cero puntos o llegar a restar I,5 ○ 2,5 puntos cuando se incluyen ideas adicionales o se llega a producir un contrasentido. 


\begin{tabular}{|c|c|c|c|c|}
\hline $\begin{array}{l}\text { Puntuación } \\
\text { Criterio }\end{array}$ & 5 & 3 & 1,5 & 0 \\
\hline Presentación & $\begin{array}{l}\text { Tono adecuado y } \\
\text { acorde al contenido de } \\
\text { lo enunciado. Claridad } \\
\text { y vocalización; } \\
\text { pronunciación } \\
\text { inteligible de todas las } \\
\text { palabras. Postura } \\
\text { correcta y relajada. } \\
\text { Transmisión r de } \\
\text { seguridad. Expresión } \\
\text { fluida, sin falsos } \\
\text { comienzos y con las } \\
\text { pausas adecuadas. }\end{array}$ & 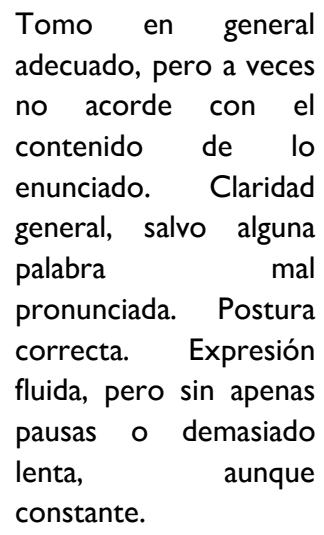 & $\begin{array}{l}\text { Tono apenas } \\
\text { adecuado. Casi todos } \\
\text { los enunciados claros, } \\
\text { pero más de una } \\
\text { palabra pral } \\
\text { pronunciada. Postura } \\
\text { poco correcta. } \\
\text { Utilización de alguna } \\
\text { muletilla, «eh» o falso } \\
\text { comienzo/ ausencia } \\
\text { total de pausas. }\end{array}$ & $\begin{array}{l}\text { Tono absolutamente } \\
\text { inapropiado. Claridad } \\
\text { prácticamente } \\
\text { ausente y más de una } \\
\text { palabra incorrecta. } \\
\text { Movimiento corporal } \\
\text { inadecuado } \\
\text { ausencia de contacto } \\
\text { visual. Fluidez escasa } \\
\text { con muletillas, «eh» } \\
\text { falsos comienzos. }\end{array}$ \\
\hline
\end{tabular}




\begin{tabular}{|l|l|l|l|l|}
\hline Expresión & $\begin{array}{l}\text { Precisión. Expresiones } \\
\text { idiomáticas acertadas. } \\
\text { Corrección. Registro } \\
\text { adecuado. }\end{array}$ & $\begin{array}{l}\text { Lengua correcta y } \\
\text { natural, ausente de } \\
\text { calcos. }\end{array}$ & $\begin{array}{l}\text { Utilización de alguna } \\
\text { expresión incorrecta } \\
\text { (calco, error } \\
\text { gramatical) o de } \\
\text { registro inapropiado. }\end{array}$ & $\begin{array}{l}\text { Empleo de más de } \\
\text { una expresión } \\
\text { incorrecta o de } \\
\text { registro inapropiado. }\end{array}$ \\
\hline $\begin{array}{l}\text { Resolución de } \\
\text { problemas }\end{array}$ & $\begin{array}{l}\text { Solución acertada (e } \\
\text { imperceptible) a las } \\
\text { dificultades } \\
\text { encontradas }\end{array}$ & $\begin{array}{l}\text { Solución rápida y } \\
\text { profesional a las } \\
\text { dificultades } \\
\text { encontradas, aunque } \\
\text { perceptible }\end{array}$ & $\begin{array}{l}\text { Solución no } \\
\text { profesional ni } \\
\text { adecuada a las } \\
\text { dificultades } \\
\text { encontradas }\end{array}$ & $\begin{array}{l}\text { Bloqueo e } \\
\text { incapacidad de } \\
\text { resolución }\end{array}$ \\
\hline
\end{tabular}




\begin{tabular}{|c|c|c|c|c|c|}
\hline \multirow[t]{3}{*}{ Contenido } & $\begin{array}{l}\text { Síntesis y } \\
\text { división } \\
\text { de la } \\
\text { atención }{ }^{1}\end{array}$ & Completo. & $\begin{array}{l}\text { Ideas fundamen-tales. } \\
\text { Ausencia de alguna idea } \\
\text { se-cundaria }\end{array}$ & $\begin{array}{l}\text { Selección mejorable } \\
\text { de la información. In- } \\
\text { completo }\end{array}$ & $\begin{array}{l}\text { Frase sílfrase no. } \\
\text { Ausencia de división } \\
\text { de la atención. El } \\
\text { contenido no se } \\
\text { corresponde en } \\
\text { absoluto con el } \\
\text { original. }\end{array}$ \\
\hline & \multirow{2}{*}{$\begin{array}{l}\text { Equiva- } \\
\text { lencia }\end{array}$} & +1 & 0 & $-1,5$ & $-2,5$ \\
\hline & & $\begin{array}{l}\text { Ausencia de ideas que } \\
\text { no están en el original, } \\
\text { salvo explicaciones } \\
\text { culturales necesarias }\end{array}$ & $\begin{array}{l}\text { Introducción de un } \\
\text { matiz no precisado en } \\
\text { el discurso original, } \\
\text { pero que no contradice } \\
\text { su sentido }\end{array}$ & $\begin{array}{l}\text { Introducción de una } \\
\text { idea ausente del } \\
\text { discurso original }\end{array}$ & $\begin{array}{l}\text { Adición de una idea } \\
\text { que contradice el } \\
\text { discurso original } \\
\text { (contrasentido) }\end{array}$ \\
\hline
\end{tabular}

Tabla I: Propuesta de matriz de valoración para evaluar la interpretación consecutiva (Domínguez Araújo, 20I3: 2I4-

$$
\text { 215). }
$$

' Es imprescindible obtener un mínimo de 2,5 en esta categoría para superar la prueba. 
Para adaptar la matriz expuesta a la situación de interpretación bilateral, hemos incluido dos áreas adicionales, que hemos denominado 'gestión del discurso' y 'expresión en el idioma extranjero'. El primer campo, la gestión del discurso, se ha incorporado a partir de las consideraciones de Jacobson (2009: 65) para su rúbrica de interpretación bilateral en el ámbito sanitario, e incorporamos la descripción de este elemento evaluador tal como queda descrito en su rúbrica, ya que tiene plena validez en el ámbito turístico: por ejemplo, si el intérprete cambia de primera a tercera persona, si el intérprete fomenta la interacción entre los interlocutores, si estos pueden acabar su turno o, por el contrario, si el intérprete interrumpe debido a una insuficiente capacidad de memoria o de toma de notas, etc. En cuanto a la asignación de puntos en ambas categorías adicionales, hemos seguido el criterio de la matriz de Domínguez Araújo (20l3).

Si bien no se ha llegado a un consenso sobre la idoneidad de emplear la primera o la tercera persona a la hora de realizar una interpretación bilateral, actuando como intérprete canal en el primer caso o como intérprete defensor, en el segundo (Aguirre Fernández Bravo y Roca Urgorri, 2015: 22), hemos considerado acertado seguir el criterio evaluador de Jacobson por el que se opta por emplear la primera persona, ya que es el criterio más aceptado al facilitar una comunicación más directa entre los interlocutores.

Por otro lado, también hemos considerado adecuado adaptar los apartados de 'presentación' y de 'expresión' descritos en la matriz de evaluación de la interpretación consecutiva, para incorporar la situación dialógica que implica la interpretación directa e inversa, es decir, desde y hacia la lengua materna, de manera alternada. En cuanto a la puntuación otorgada, hemos mantenido el criterio de puntuación de 0 a 5 de Domínguez Araújo (2013).

A continuación, presentamos la rúbrica específica para la evaluación de interpretaciones bilaterales en el ámbito del turismo, con las adaptaciones descritas: 


\begin{tabular}{|c|c|c|c|c|}
\hline $\begin{array}{l}\text { Puntuación } \\
\text { Criterio }\end{array}$ & 5 & 3 & 1,5 & 0 \\
\hline Presentación & $\begin{array}{l}\text { Tono adecuado y acorde } \\
\text { al contenido de lo } \\
\text { enunciado. Claridad y } \\
\text { vocalización; } \\
\text { pronunciación inteligible } \\
\text { de todas las palabras. } \\
\text { Postura correcta y } \\
\text { relajada. Transmisión de } \\
\text { seguridad. Expresión } \\
\text { fluida, sin falsos } \\
\text { comienzos y con las } \\
\text { pausas adecuadas. }\end{array}$ & $\begin{array}{l}\text { Tomo en general } \\
\text { adecuado, pero a veces } \\
\text { no acorde con el } \\
\text { contenido de lo } \\
\text { enunciado. Claridad } \\
\text { general, salvo alguna } \\
\text { palabra mal pronunciada. } \\
\text { Postura correcta. } \\
\text { Expresión fluida, pero } \\
\text { sin apenas pausas o } \\
\text { demasiado lenta, aunque } \\
\text { constante. }\end{array}$ & $\begin{array}{l}\text { Tono apenas } \\
\text { adecuado. Casi } \\
\text { todos los enunciados } \\
\text { claros, pero más de } \\
\text { una palabra mal } \\
\text { pronunciada. Postura } \\
\text { poco correcta. } \\
\text { Utilización de alguna } \\
\text { muletilla, «eh» o } \\
\text { falso comienzo/ } \\
\text { ausencia total de } \\
\text { pausas. }\end{array}$ & $\begin{array}{l}\text { Tono absolutamente } \\
\text { inapropiado. Claridad } \\
\text { prácticamente ausente } \\
\text { y más de una palabra } \\
\text { incorrecta. Movimiento } \\
\text { corporal inadecuado o } \\
\text { ausencia de contacto } \\
\text { visual. Fluidez escasa } \\
\text { con muletillas, «eh» o } \\
\text { falsos comienzos. }\end{array}$ \\
\hline $\begin{array}{l}\text { Gestión } \\
\text { discurso }\end{array}$ & $\begin{array}{l}\text { Uso consistente de la } \\
\text { primera persona cam- } \\
\text { biando a la tercera } \\
\text { persona solo para aclarar; } \\
\text { fomenta la interacción, } \\
\text { incluyendo contacto visual } \\
\text { entre los interlocutores, }\end{array}$ & $\begin{array}{l}\text { Uso consistente de la } \\
\text { primera persona, } \\
\text { cambiando a la tercera } \\
\text { persona solo para } \\
\text { aclarar; fomenta la } \\
\text { interacción en general, } \\
\text { incluyendo contacto }\end{array}$ & $\begin{array}{l}\text { Uso inconsistente de } \\
\text { la primera persona; } \\
\text { uso excesivo de la } \\
\text { tercera persona } \\
\text { creando } \\
\text { incomodidad; casi no } \\
\text { fomenta la }\end{array}$ & $\begin{array}{l}\text { Uso inconsistente de la } \\
\text { primera persona; uso } \\
\text { casi excesivo de la } \\
\text { tercera persona } \\
\text { creando incomodidad; } \\
\text { no fomenta la inter- } \\
\text { acción entre los }\end{array}$ \\
\hline
\end{tabular}




\begin{tabular}{|c|c|c|c|c|}
\hline & $\begin{array}{l}\text { tanto verbalmente como a } \\
\text { través de otras señales } \\
\text { paralingüísticas; permite a } \\
\text { los interlocutores } \\
\text { terminar su turno gracias } \\
\text { a una buena capacidad de } \\
\text { memorización y una } \\
\text { buena habilidad de toma } \\
\text { de notas; demuestra } \\
\text { estrategias efectivas para } \\
\text { manejar solapamientos. }\end{array}$ & $\begin{array}{l}\text { visual entre los } \\
\text { interlocutores, tanto } \\
\text { verbalmente como a } \\
\text { través de otras señales } \\
\text { paralingüísticas; en } \\
\text { general permite a los } \\
\text { interlocutores terminar } \\
\text { su turno sin interrumpir } \\
\text { para aclaraciones; cierta } \\
\text { dificultad debido a la } \\
\text { necesidad de seguir } \\
\text { desarrollando la } \\
\text { memoria y la habilidad } \\
\text { de tomar notas, así } \\
\text { como mejorar el } \\
\text { vocabulario. En general } \\
\text { maneja solapamientos } \\
\text { con calma y eficiencia, } \\
\text { pero demuestra la } \\
\text { necesidad de seguir } \\
\text { practicando. }\end{array}$ & $\begin{array}{l}\text { interacción } r \\
\text { general, ni ver- } \\
\text { balmente ni a través } \\
\text { de otras señales } \\
\text { paralingüísticas; inte- } \\
\text { rrumpe a los } \\
\text { interlocutores a } \\
\text { menudo para pedir } \\
\text { aclaraciones debido } \\
\text { a la necesidad de } \\
\text { desarrollar la } \\
\text { memoria y la toma } \\
\text { de notas en general; } \\
\text { permite a los } \\
\text { interlocutores } \\
\text { terminar su turno } \\
\text { sin interrumpir para } \\
\text { aclaraciones; cierta } \\
\text { dificultad debido a la } \\
\text { necesidad de seguir } \\
\text { desarrollando la la } \\
\text { memoria y una toma } \\
\text { de notas así como }\end{array}$ & 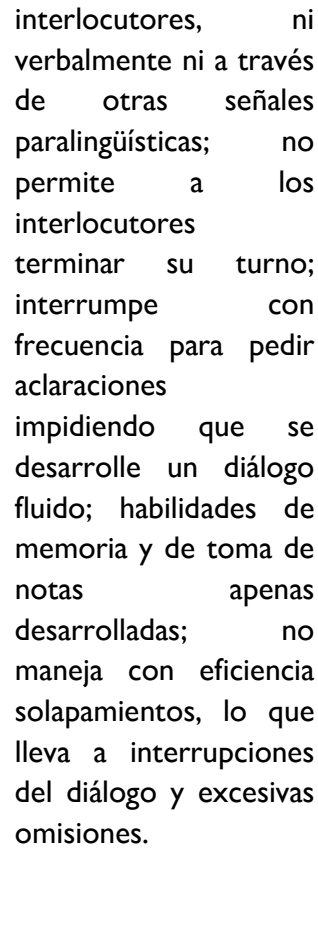 \\
\hline
\end{tabular}




\begin{tabular}{|c|c|c|c|c|}
\hline & & & $\begin{array}{lr}\text { mejorar } & \text { el } \\
\text { vocabulario. Se pone } \\
\text { nervioso/a con } \\
\text { solapamientos } \\
\text { demostrando una } \\
\text { necesidad de } \\
\text { practicando. }\end{array}$ & \\
\hline $\begin{array}{l}\text { Expresión en la } \\
\text { lengua materna }\end{array}$ & $\begin{array}{l}\begin{array}{l}\text { Precisión. } \\
\text { Expresiones } \\
\text { idiomáticas } \\
\text { Corrección. }\end{array} \text { Registro } \\
\text { adecuado. Tono adecuado } \\
\text { y acorde al contenido de } \\
\text { lo enunciado. Claridad y } \\
\text { vocalización; Expresión } \\
\text { fluida, sin falsos } \\
\text { comienzos y con las } \\
\text { pausas adecuadas. }\end{array}$ & $\begin{array}{l}\text { Lengua correcta y } \\
\text { natural, ausente de } \\
\text { calcos. Tono en general } \\
\text { adecuado, pero a veces } \\
\text { no acorde con el } \\
\text { contenido de lo } \\
\text { enunciado. Claridad } \\
\text { general. Expresión } \\
\text { demasiado lenta, aunque } \\
\text { constante. Utilización de } \\
\text { alguna muletilla, "eh". }\end{array}$ & $\begin{array}{l}\text { Utilización de alguna } \\
\text { expresión incorrecta } \\
\text { (calco, error } \\
\text { gramatical) o de } \\
\text { registro inapropiado. } \\
\text { Tono apenas } \\
\text { adecuado. Muchos } \\
\text { de los enunciados no } \\
\text { son claros. Postura } \\
\text { poco correcta. } \\
\text { Utilización "eh" o } \\
\text { muletillas, "eh" } \\
\text { falsos comienzos. }\end{array}$ & $\begin{array}{l}\text { Empleo de más de una } \\
\text { expresión incorrecta o } \\
\text { de registro } \\
\text { inapropiado. Tono } \\
\text { absolutamente } \\
\text { inapropiado. Claridad } \\
\text { prácticamente ausente. } \\
\text { Fluidez escasa con gran } \\
\text { cantidad de muletillas, } \\
\text { "eh" o falsos } \\
\text { comienzos. }\end{array}$ \\
\hline
\end{tabular}




\begin{tabular}{|c|c|c|c|c|}
\hline $\begin{array}{l}\text { Expresión en la } \\
\text { lengua extranjera }^{2}\end{array}$ & $\begin{array}{l}\text { Expresiones idiomáticas } \\
\text { acertadas. Casi no comete } \\
\text { errores gramaticales o de } \\
\text { pronunciación. Registro } \\
\text { adecuado. Tono adecuado } \\
\text { y acorde al contenido de } \\
\text { lo enunciado. Claridad y } \\
\text { vocalización; Expresión } \\
\text { fluida, sin falsos } \\
\text { comienzos y con las } \\
\text { pausas adecuadas. Muy } \\
\text { pocas o ninguna muletilla, } \\
\text { "eh". }\end{array}$ & $\begin{array}{l}\text { Lengua correcta, en } \\
\text { general no comete } \\
\text { muchos errores } \\
\text { gramaticales o de pro- } \\
\text { nunciación. Registro en } \\
\text { general adecuado. Tono } \\
\text { en general adecuado, } \\
\text { pero a veces no acorde } \\
\text { con el contenido de lo } \\
\text { enunciado. Claridad } \\
\text { general. Expresión } \\
\text { demasiado lenta, aunque } \\
\text { constante. Utilización de } \\
\text { alguna muletilla, "eh". }\end{array}$ & $\begin{array}{l}\text { Expresiones } \\
\text { incorrectas. Comete } \\
\text { errores gramaticales } \\
\text { o de pronunciación } \\
\text { con frecuencia. } \\
\text { Registro a menudo } \\
\text { no adecuado. Tono } \\
\text { apenas adecuado. } \\
\text { Muchos de los } \\
\text { enunciados no son } \\
\text { claros Postura poco } \\
\text { correcta. Utilización } \\
\text { de muletillas, "eh" o } \\
\text { falsos comienzos. }\end{array}$ & $\begin{array}{l}\text { No se entiende. } \\
\text { Multitud de errores } \\
\text { gramaticales y de } \\
\text { pronunciación. Pausas } \\
\text { largas, expresiones } \\
\text { incorrectas, registro no } \\
\text { adecuado. Tono } \\
\text { absolutamente } \\
\text { inapropiado. Claridad } \\
\text { prácticamente ausente. } \\
\text { Fluidez escasa con gran } \\
\text { cantidad de muletillas, } \\
\text { "eh" o falsos } \\
\text { comienzos. }\end{array}$ \\
\hline $\begin{array}{l}\text { Resolución } \\
\text { problemas }\end{array}$ & $\begin{array}{l}\text { Solución acertada (e } \\
\text { imperceptible) a las } \\
\text { dificultades encontradas. }\end{array}$ & $\begin{array}{lcc}\text { Solución } & \text { rápida } & \text { y } \\
\text { profesional } & \text { a } & \text { las } \\
\text { dificultades encontradas, } \\
\text { aunque perceptible. }\end{array}$ & $\begin{array}{lrr}\text { Solución } & & \text { no } \\
\text { profesional } & & \text { ni } \\
\text { adecuada a } & \text { las } \\
\text { dificultades } & & \\
\text { encontradas. } & & \end{array}$ & $\begin{array}{l}\text { Bloqueo e incapacidad } \\
\text { de resolución. }\end{array}$ \\
\hline
\end{tabular}

${ }^{2}$ Es imprescindible obtener un mínimo de 2,5 en esta categoría para superar la prueba 


\begin{tabular}{|c|c|c|c|c|c|}
\hline \multirow[t]{3}{*}{$\begin{array}{l}\text { Conte- } \\
\text { nido }\end{array}$} & $\begin{array}{l}\text { Síntesis y } \\
\text { división } \\
\text { de la } \\
\text { atención }^{3}\end{array}$ & Completo. & $\begin{array}{l}\text { Ideas fundamentales. } \\
\text { Ausencia de alguna idea } \\
\text { secundaria. }\end{array}$ & $\begin{array}{l}\text { Selección mejorable } \\
\text { de la información; in- } \\
\text { completo. }\end{array}$ & $\begin{array}{l}\text { Frase sí/frase no. } \\
\text { Ausencia de división de } \\
\text { atención. El contenido } \\
\text { no se corresponde en } \\
\text { absoluto con el } \\
\text { original. }\end{array}$ \\
\hline & \multirow{2}{*}{$\begin{array}{l}\text { Equiva- } \\
\text { lencia }\end{array}$} & +1 & 0 & $-1,5$ & $-2,5$ \\
\hline & & $\begin{array}{l}\text { Ausencia de ideas que no } \\
\text { están en el original, salvo } \\
\text { explicaciones culturales } \\
\text { necesarias. }\end{array}$ & $\begin{array}{l}\text { Introducción de un matiz } \\
\text { no precisado en el } \\
\text { discurso original, pero } \\
\text { que no contradice su } \\
\text { sentido. }\end{array}$ & $\begin{array}{l}\text { Introducción de una } \\
\text { idea ausente del } \\
\text { discurso original. }\end{array}$ & $\begin{array}{l}\text { Adición de una idea } \\
\text { que contradice el } \\
\text { discurso original } \\
\text { (contrasentido). }\end{array}$ \\
\hline
\end{tabular}

Tabla 2: Tabla adaptada para la evaluación de interpretaciones bilaterales

\footnotetext{
${ }^{3}$ Es imprescindible obtener un mínimo de 2,5 en esta categoría para superar la prueba.
} 


\section{Descripción del corpus}

Para llevar a cabo el análisis, hemos creado un corpus bilingüe (alemánespañol) que hemos denominado CIBITU (Corpus de Interpretaciones Bilaterales en Turismo), compuesto por interpretaciones bilaterales en el ámbito del turismo, producidas por nuestros alumnos de último curso en el examen final de la asignatura. Para este objetivo, fue indispensable integrar una colección de interpretaciones bilaterales recogidas en las evaluaciones finales, que se codificaron con la intención de facilitar el registro posterior de los análisis y los resultados. De esta manera, puede clasificarse como corpus oral, por estar compuesto por grabaciones en las que se recogen interpretaciones bilaterales.

Con este corpus, hemos formado una colección de elementos lingüísticos bilingües que conforman la muestra a analizar con la intención de alcanzar conclusiones sobre la tipología de errores, su frecuencia y la posible variación por direccionalidad, que nos ayuden a perfilar propuestas didácticas tendentes a mejorar la producción.

Las producciones se grabaron en formato digital y se han recopilado durante los cursos académicos 2016/2017 y 2017/2018, en las dos convocatorias oficiales de examen de cada curso. Todos los participantes en los dos cursos analizados son nativos de español, excepto una alumna en el curso 2016/2017, nativa de alemán, que disfrutaba de una beca de movilidad Erasmus y que se presentó en la primera convocatoria, obteniendo una calificación superior a 5 sobre 10 puntos.

El corpus CIBITU es una muestra actual que está compuesta por grabaciones digitales de interpretación bilaterales con una duración que varía entre los 9 y los 10,5 minutos. Las interpretaciones se distribuyen de la siguiente manera: del curso 2016/2017, se incluyen 6 grabaciones correspondientes a las evaluaciones de la primera convocatoria, en mayo de 2017, y I grabación de la segunda convocatoria, en junio del mismo año; del curso 2017/2018, se incluyen 5 grabaciones de la evaluación en la primera convocatoria, en mayo de 2018, así como 2 grabaciones en la segunda convocatoria de junio de 2018. En total, suman 14 grabaciones.

Torruella y Llisterri (1999: I5 y ss.) establecen los aspectos esenciales a tener en cuenta en el diseño de un corpus:

a) Finalidad: el corpus CIBITU se crea con la intención de recopilar interpretaciones bilaterales en el ámbito del turismo, producidas por nuestros alumnos de último curso, representativas de esta área de especialidad, para realizar un análisis detallado sobre los tipos de errores y su frecuencia, así como sobre sus implicaciones en la didáctica. 
b) Límite: la recopilación de grabaciones se llevó a cabo durante dos cursos consecutivos, en la primera convocatoria de evaluación, en mayo de cada año, y en la segunda convocatoria de evaluación, en junio. Corresponden a alumnos de la misma asignatura, impartida durante los dos años por el mismo equipo docente y con la misma secuenciación de contenidos.

c) Tipo: oral, con una muestra representativa de producciones relacionadas con la interpretación bilateral en el turismo, que toman como base un diálogo que simula la realidad, creado expresamente para cada examen.

d) Población y muestra: 14 alumnos de último curso en 2016/2017 y $2017 / 2018$

e) Número y longitud de la muestra: 14 grabaciones distribuidas en dos cursos académicos consecutivos, con una duración que varía entre 9 y 10,5 minutos.

f) En relación a la obtención de los textos, se realizaron grabaciones de las producciones de los alumnos, con su consentimiento, a través de un procedimiento digital.

g) En cuanto al procesamiento posterior, dado que se partía de un documento escrito ${ }^{4}$, elaborado de manera previa a la realización de la interpretación, se realizaron anotaciones en el documento original, estableciendo un etiquetado de elementos de distorsión a partir de la clasificación de errores establecida.

\section{Resultados del análisis}

A continuación, presentamos los resultados más relevantes alcanzados tras el análisis del corpus CIBITU. La siguiente tabla muestra el número de estudiantes que han obtenido cada puntuación en cada uno de los elementos evaluativos de la matriz.

\footnotetext{
4 Aun cuando para realizar el examen se parte de un guion, las profesoras participantes en la escenificación del diálogo realizan una interacción flexible, donde se evita la lectura, con aportaciones espontáneas, que dependen del alumno y su producción, para acercarnos lo más posible a la situación real.
} 


\begin{tabular}{|c|c|c|c|c|}
\hline & 5 puntos & 3 puntos & 1,5 puntos & $\begin{array}{c}0 \\
\text { puntos }\end{array}$ \\
\hline $\begin{array}{l}\text { Presentación (para la } \\
\text { lengua materna) }\end{array}$ & 4 & 6 & 4 & \\
\hline Gestión del discurso & 6 & 5 & 2 & 1 \\
\hline $\begin{array}{l}\text { Expresión en la lengua } \\
\text { materna }\end{array}$ & 7 & 4 & 3 & \\
\hline $\begin{array}{l}\text { Expresión en la lengua } \\
\text { extranjera }\end{array}$ & 2 & 3 & 9 & \\
\hline Resolución de problemas & 2 & 9 & 3 & \\
\hline \multirow{2}{*}{$\begin{array}{l}\text { Síntesis y división de la } \\
\text { atención }\end{array}$} & 1 & 8 & 3 & 2 \\
\hline & +1 puntos & 0 puntos & $-1,5$ puntos & $\begin{array}{c}-2,5 \\
\text { puntos }\end{array}$ \\
\hline Equivalencia & 6 & 6 & 1 & 1 \\
\hline
\end{tabular}

Tabla 3: Distribución de los alumnos según la puntuación obtenida en cada elemento evaluativo.

Como se puede observar en la tabla 3, los estudiantes muestran un nivel adecuado en las interpretaciones producidas en los exámenes de evaluación de la asignatura, especialmente en las áreas de resolución de problemas, expresión en la lengua materna y síntesis y división de la atención. El siguiente gráfico muestra los resultados de una manera más clara:

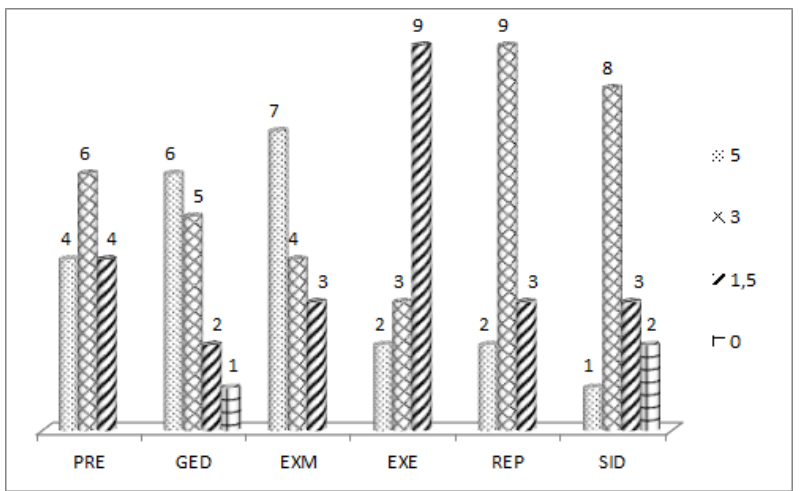

Gráfico I: Distribución de alumnos por puntuación en cada elemento evaluativo de la matriz

En los datos recogidos en la tabla 3 y en el gráfico I, observamos que el elemento en el que un mayor número de estudiantes logra la máxima calificación es 
la expresión en lengua materna, pues II alumnos lograron 3 ○ 5 puntos, de un máximo de 5 , mientras que los tres estudiantes restantes alcanzaron una puntuación de I,5. El hecho de que varios estudiantes no alcancen una puntuación adecuada al expresarse en su propia lengua materna constata la necesidad de realizar prácticas formativas que también tengan en cuenta este elemento, ya que la expresión en el idioma nativo puede verse afectada por debilidad en otras capacidades necesarias, como pueden ser la memoria, la técnica de toma de notas o la gestión del discurso (Gile 2009: 157-190; Abril Martí y Collados Aís 2001: 95127).

Comparándolo con la puntuación de la expresión en lengua extranjera, observamos un empeoramiento a tener en cuenta, pues aunque 5 estudiantes logran 3 o 5 puntos, 9 consiguen I,5, lo que indica que es un aspecto que necesita trabajarse de manera más eficaz en el aula.

Hemos llevado a cabo una categorización de los errores detectados en las producciones evaluadas, de tal manera que se han identificado los siguientes tipos, agrupados en tres aspectos fundamentales:

a) Forma: interferencias del inglés (INI), cambios de registro (RGE), tratamiento inadecuado de referencias culturales (RFN), tratamiento inadecuado de principios de intervención (PIO),

b) Contenido: faltas de comprensión (CDS), adición no necesaria ( $A D I$ ), omisiones (que hemos diferenciado entre relevantes, OMR, leves, OML, O no significativas $\mathrm{OMN}$ ),

c) Gestión de la comunicación: interrupciones inadecuadas del discurso de los intervinientes (FCP), pausas en la expresión en lengua extranjera (PAU), fluidez (FLU).

El siguiente gráfico muestra los errores detectados más relevantes por su frecuencia de aparición: 


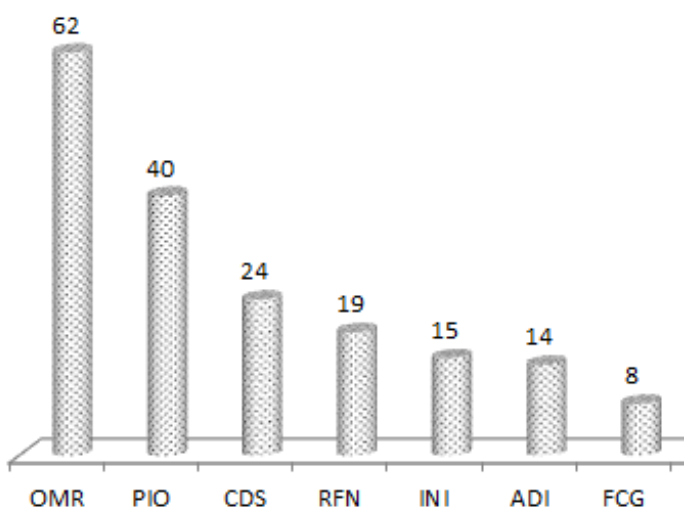

Gráfico 2: Principales errores detectados

Como se puede observar en el gráfico 2, los estudiantes principalmente cometen omisiones relevantes de la información contenida en el mensaje original de cualquiera de los dos intervinientes en el diálogo, lo que puede producir una pérdida de la información emitida por el agente turístico o bien de las necesidades expresadas por el turista, conllevando, por tanto, la producción de una interpretación no adecuada.

De igual manera, hemos observado que con frecuencia se produce un tipo de omisión que hemos considerado adecuado analizar de manera independiente. Se trata de los principios de intervención de cada interlocutor en el diálogo interpretado, que no suelen transmitir mucha información, sino más bien aportan un tono específico al diálogo y además proporcionan cohesión interna en el discurso, ya que suelen expresar las emociones producidas por la información recibida (como alegría, sorpresa, agrado, tristeza, desagrado, confirmación de comprensión, etc.). La omisión de la expresión de estas emociones conlleva la pérdida parcial de la intención comunicativa, por lo que debe tenerse en cuenta a la hora de evaluar la calidad de la interpretación bilateral.

Otras categorías a tener en cuenta por su frecuencia de aparición son los cambios de sentido; las referencias culturales no explicitadas, que pueden producir cierta confusión en el turista; la influencia del inglés, por la que se emplean términos en inglés a la hora de producir las expresiones en alemán; así como las adiciones no necesarias, es decir, aquellas que no están destinadas a ayudar en la comprensión del mensaje o explicitar en la cultura meta información implícita en la cultura origen, sino que sirven, por ejemplo, para dar una opinión. 
Debemos destacar, en relación a dos de las categorías expuestas, que existe una mayor frecuencia de casos en los que los estudiantes han sabido gestionarlas. Concretamente, nos referimos a los principios de intervención, que se han trasladado en 56 ocasiones (frente a las 40 en las que no se han trasladado), produciendo cohesión intradiscursiva, así como las referencias culturales explicadas (33 casos frente a 19 no explicitadas).

En cuanto a las interrupciones inadecuadas del diálogo que se está interpretando, nos referimos al empleo de alguna fórmula de cortesía para interrumpir al interviniente en el diálogo para solicitar una aclaración o precisar algo que no se haya comprendido o escuchado. Como se puede observar en el gráfico 3 , de los alumnos que hacen este tipo de preguntas a los intervinientes en el diálogo, una amplia mayoría no emplea fórmulas de cortesía.

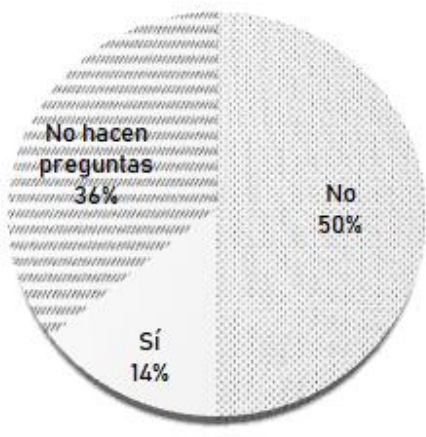

Gráfico 3: Fórmulas de cortesía en interrupciones

Profundizando en el aspecto de la expresión en lengua extranjera, concretamente en alemán, hemos categorizado los errores en cuatro bloques principales, tal como se muestra en el gráfico 4: léxico (LEX), expresiones (EXP), cambios de intensidad en el discurso (CID) y gramática (GRA). El criterio 'cambio de intensidad en el discurso' se refiere a la interpretación que modifica el tono del interviniente, es decir, interpretar con un tono de euforia el tono serio o neutro, lo que puede producir desconcierto e incluso alteración en la recepción del mensaje interpretado. ${ }^{5}$ En cuanto a la categoría gramatical, hemos identificado, a su vez, los siguientes elementos: género del sustantivo (GEN); declinaciones (DEC); errores en el uso de verbos, como la construcción, conjugación, etc. (VER); uso de

${ }^{5}$ En este sentido, nos hemos encontrado principalmente con estudiantes que transmiten un tono de desgana y apagado, con tendencia a disminuir el volumen a medida que acaba la interpretación, aun cuando el tono del mensaje a interpretar expresaba alegría y volumen uniforme. 
preposiciones (PRE); ausencia de sujeto (ASU); errores en números, fechas, horas, etc. (NUM); errores en la concordancia sujeto-verbo (CON); errores en oraciones subordinadas (OSU); errores en el orden de los elementos en la oración (ORD); y una última categoría de otros (OTR).

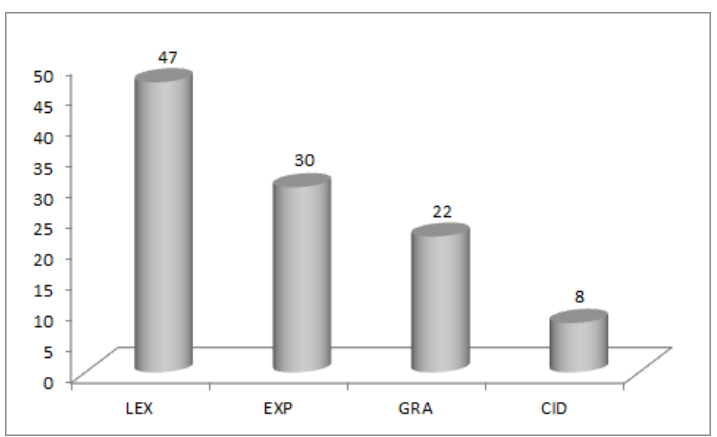

Gráfico 4: Frecuencia de aparición de errores en lengua extranjera

Podemos observar que los estudiantes cometen principalmente errores relacionados con el léxico y con las expresiones en lengua extranjera, mientras que los errores gramaticales se sitúan en tercer lugar. Es destacable el hecho de que los cambios de intensidad en el discurso se producen en raras ocasiones, es decir, no es habitual que el estudiante omita el tono del discurso original (por ejemplo, si el interviniente expresa alguna emoción o varias en una misma intervención y el intérprete emplea un tono neutro, omitiendo las diversas emociones expresadas en el original).

Por último, en el gráfico 5 , se presentan los errores gramaticales más relevantes:

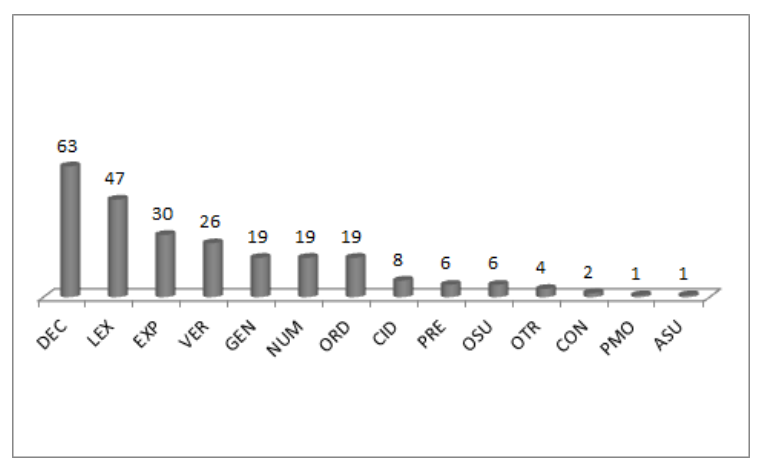

Gráfico 5: Categorización de los errores gramaticales más relevantes 
Como podemos observar, los principales problemas se concentran en el empleo de las declinaciones, de gran dificultad para los no hablantes de alemán, así como en el empleo de los verbos, el género del sustantivo, el orden de los elementos en la oración o el empleo de cifras, años o fechas.

\section{Conclusiones}

Podemos comprobar que la matriz de evaluación de la interpretación bilateral, que hemos elaborado a partir de la matriz diseñada para la interpretación consecutiva, nos ha permitido evaluar los exámenes del ámbito turístico incluidos en el corpus CIBITU, analizando para cada uno de ellos los parámetros de evaluación descritos. De esta manera, nos ha permitido detectar los errores que los alumnos cometen con mayor frecuencia a la hora de llevar a cabo las interpretaciones bilaterales de examen en el ámbito del turismo, así como aquellos elementos en los que se observa un desempeño adecuado. Estos resultados nos van a permitir desarrollar propuestas didácticas que se pondrán en práctica en el transcurso de la asignatura, en el segundo cuatrimestre del año académico.

Debido al carácter descriptivo de la matriz, en la que no solo se asigna una puntuación a la producción, sino que se describen con detalle los puntos que deben tenerse en cuenta para cada una de las áreas evaluadas, puede ser empleada en el aula, por un lado, para ofrecer al alumnado los criterios que se tendrán en cuenta a la hora de evaluar los exámenes, potenciando la transparencia en la evaluación, y por otro lado para proporcionar a los estudiantes una herramienta de autoevaluación que les facilite la práctica autónoma de la asignatura, de manera complementaria a las actividades presenciales, y que les permita conocer el punto en el que se encuentran en el proceso de aprendizaje, así como los elementos que necesitan mayor esfuerzo para conseguir una mejora y los elementos en los que alcanzan un desempeño positivo.

Las estrategias didácticas a desarrollar se pondrán en práctica, como se ha mencionado, en el trascurso de la asignatura en el segundo cuatrimestre del curso próximo e irán encaminadas a potenciar los aspectos que con mayor frecuencia provocan una dificultad para los alumnos, sin olvidar los aspectos en los que no se suelen detectar errores, y su idoneidad será evaluada cuando comparemos los resultados de los exámenes finales con los resultados del análisis expuesto en estas páginas.

Como se ha detectado en el análisis, debemos poner especial hincapié en la expresión en la lengua extranjera, a través de actividades complementarias que aporten fluidez en el dominio del idioma, concretamente: 
a) Para mejorar el uso de declinaciones, el género del sustantivo o el empleo correcto de verbos, partiremos de ejercicios de sinonimia y antonimia, poniendo énfasis en el artículo y en las declinaciones en oraciones determinadas: los estudiantes tendrán que sustituir términos en diversas oraciones, empleando sinónimos y antónimos, con lo que tendrán que decidir si deben cambiar o mantener la declinación según si el género cambia. En estos ejercicios se partirá de vocabulario general y se irá profundizando a lo largo del semestre con términos que se empleen con más frecuencia en los ámbitos de especialidad estudiados.

b) De igual manera, para mejorar el uso de las declinaciones, los verbos y el género, los estudiantes deberán completar de manera oral un texto con espacios en blanco, en los que tendrán que incluir verbos, artículos o solo la declinación. Se trataría de una actividad a realizar en casa, por lo que los estudiantes grabarían su actuación con la intención de poderla corregir posteriormente en clase, a través de comentarios sobre los resultados obtenidos por cada uno de ellos y para aclarar dudas.

c) Para mejorar la sintaxis en alemán, nos proponemos hacer uso de los ejercicios de traducción inversa a vista, que los estudiantes podrían grabar, para posteriormente repasar el orden de los elementos en la oración y poder incidir en aquellas oraciones en las que la sintaxis no sea adecuada.

d) Como actividad tendente también a la mejora en la producción oral en lengua alemana, indicaremos a los alumnos que, tras realizar la actividad del texto con espacios en blanco comentado en el segundo punto, deben grabarse hablando en alemán sobre el mismo tema, es decir, tendrían que realizar una producción de unos 2 minutos en la que expresasen una opinión o una crítica sobre la temática tratada en el texto.

No obstante, no podemos olvidar la necesidad de potenciar las técnicas complementarias, como la toma de notas o la memorización, para mejorar la expresión en la lengua materna. De igual modo, las estrategias didácticas deben tener en cuenta las interacciones de los estudiantes con los intervinientes en el discurso y deberán servir para que los estudiantes tomen conciencia de las omisiones de información relevante.

\section{Referencias bibliográficas}

Abril Martí, María I. y Collados Aís, Ángela (200I). «El modelo de esfuerzos adaptado a la didáctica de la interpretación bilateral». En Manual de 
interpretación bilateral, Collados Aís, Angela y María I. Abril Martí (coords.). Granada: Comares, 95-127.

Alcón Latorre, Mireia. 2016. «La rúbrica como instrumento de evaluación en los estudios universitarios». En Observar IO(I). I-I5.

Aguirre Fernández Bravo, Elena y Ana María Roca Urgorri. 2015. «Perfiles y roles del intérprete en los servicios públicos: el intérprete mediador como modelo de compromiso». En Investigación emergente en Traducción e Interpretación, Raquel Lázaro Gutiérrez, María del Mar Sánchez Ramos y Francisco Javier Vigier Moreno (eds.). Granada: Comares, 19-34.

Aguirre Fernández Bravo, Elena. 2016. «El uso de rúbricas en la evaluación formativa de la competencia interpretativa=Using rubrics in the formative assessment of students' interpreting competence». En Traducción e interpretación en los servicios públicos $(\mathrm{TISP})=$ Public service interpreting and translation (PIST) formación, evaluación y acreditación = training, testing and accreditation, Carmen Valero Garcés (coord.), Bianca Vitalaru (col.) y Raquel Obama Oboam (col.). Alcalá de Henares: Servicio de Publicaciones de la Universidad de Alcalá de Henares, I-I5.

Arter, Judith y Jay McTighe. 200I. Scoring rubrics in the classroom: Using performance criteria for assessing and improving student performance, Thousand Oaks, CA: Corwin/Sage.

Cirillo, Letizia y Natacha Niemants. 2017. Teaching Dialogue Interpreting, Amsterdam: John Benjamins.

Collados Aís, Ángela y María Manuela Fernández Sánchez. 200I. «La interpretación bilateral: características, situaciones comunicativas y modalidades». En Manual de interpretación bilateral, Collados Aís, Ángela y María Manuela Fernández Sánchez (coords.). Granada: Comares, 6I-77.

Domínguez Araújo, Lara. 2013. «Una propuesta de matriz formativa para evaluar la interpretación consecutiva». En Quality Interpreting: Widening the scope, vol. 2, Rafael Barranco-Droege, E. Macarena Pradas Macías y Olalla García Becerra (eds). Granada: Comares (Interlingua), 20I-222.

Gile, Daniel. 2009. Basic Concepts and Models for Interpreter and Translator Training. Amsterdam / Philadelphia: John Benjamins.

Jacobson, Holly E. 2009. «Moving beyond words in assessing mediated interaction». En Testing and assessment in Translation and Interpreting Studies, Claudia V. Angelelli y Holly E. Jacobson (eds.). Amsterdam / Philadelphia: John Benjamins, 49-69.

Reddy, Y. Malini y Heidi Andrade. 2009. «A review of rubric use in higher education». En Assessment \& evaluation in higher education 35(4), 435-448.

Ruiz Mezcua, Aurora. 2010. "La enseñanza de la interpretación social en el contexto biosanitario: la preparación psicológica del intérprete médico». En Trans 14. 143-160. 
Stiggins, Richard J. 200I. Student-involved classroom assessment. $3^{\mathrm{a}}$ ed., Upper Saddle River, NJ: Prentice-Hall.

Torruella, Joan y Joaquim Llisterri 1999. «Diseño de corpus textuales y orales». En José Manuel Blecua, Gloria Clavería, Carlos Sánchez y Joan Torruella (eds.) Filología e informática: Nuevas tecnologías en los estudios filológicos. Barcelona: Seminario de Filología e Informática, Departamento de Filología Española, Universidad Autónoma de Barcelona. Editorial Milenio, 45-77. 